\title{
High Gain Preamplifier Module (PAM) Engineering Prototype for the National Ignition Facility (NIF) Laser System
}

M. D. Martinez, K. M. Skulina, F. J. Deadrick,

J. Braucht, B. Jones, S. Hawkins,

R. Tilley, R. Wing, J. Crawford,

D. Browning, J. K. Crane, and F. Penko

This paper was prepared for submittal to the

Third Annual International Conference on

Solid State Lasers for Application (SSLA)

to Inertial Confinement Fusion (ICF)

Monterey, California

June 7-12, 1998

October 29, 1998

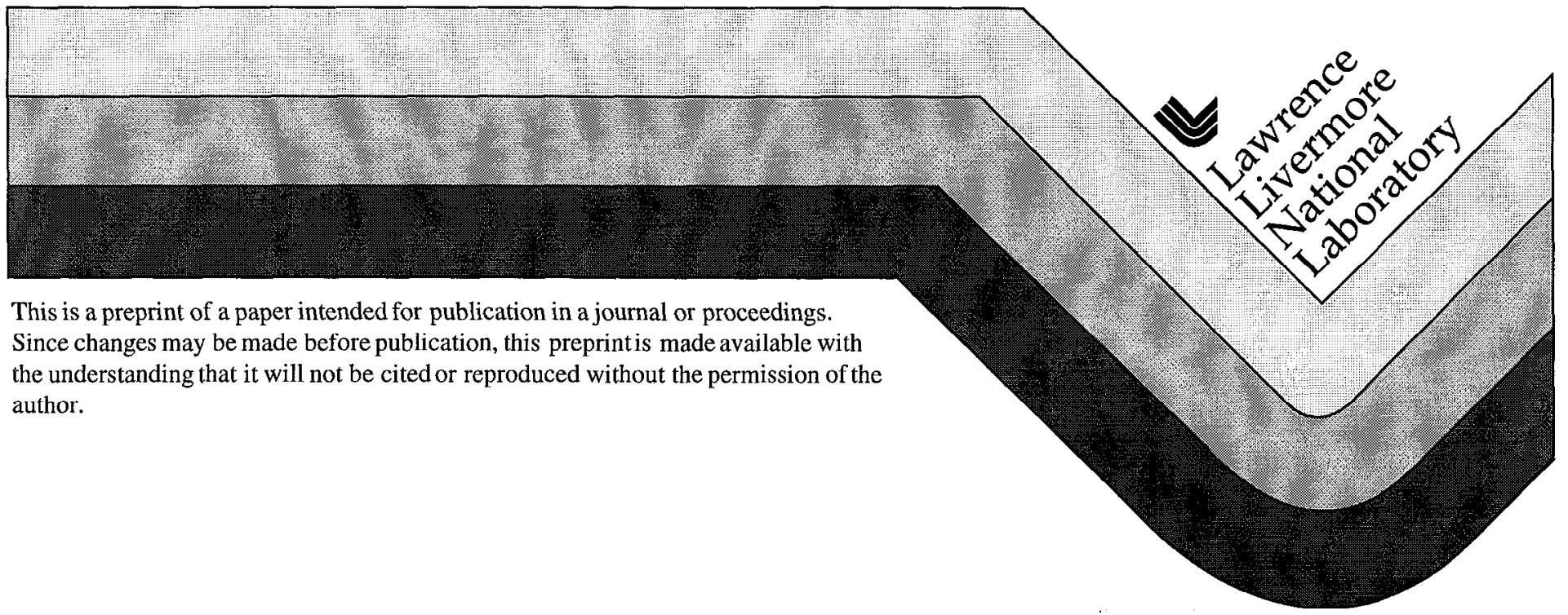




\section{DISCLAIMER}

This document was prepared as an account of work sponsored by an agency of the United States Government. Neither the United States Government nor the University of California nor any of their employees, makes any warranty, express or implied, or assumes any legal liability or responsibility for the accuracy, completeness, or usefulness of any information, apparatus, product, or process disclosed, or represents that its use would not infringe privately owned rights. Reference herein to any specific commercial product, process, or service by trade name, trademark, manufacturer, or otherwise, does not necessarily constitute or imply its endorsement, recommendation, or favoring by the United States Government or the University of California. The views and opinions of authors expressed herein do not necessarily state or reflect those of the United States Government or the University of California, and shall not be used for advertising or product endorsement purposes. 
High gain Preamplifier Module (PAM) engineering prototype for the National Ignition Facility (NIF) laser system

Mikael D. Martinez, K.M. Skulina, F.J. Deadrick, J. Braucht, B. Jones, S. Hawkins, R. Tilley, R.Wing, J. Crawford, D. Browning, J.K. Crane and F. Penko

Lawrence Livermore National Laboratory

M.S. L-483 7000 E. Ave

Livermore, CA 94550

\begin{abstract}
We describe recent results and developments in the preamplifier module (PAM) engineering prototype located in NIF's front end or Optical Pulse Generation (OPG) system. This prototype uses the general laser design developed on a physics testbed ${ }^{(1,2)}$ and integrates NIF type packaging as well as controls and diagnostics. We will present laser, mechanical and electrical hardware designed and built to date as well as laser energetics measurements.
\end{abstract}

Keywords: NIF, Nd:Glass, diode pumped, pre-amplifier, SSD

\title{
1.0 INTRODUCTION
}

The NIF front end or OPG consists of an all fiber-based master oscillator ${ }^{(3)}$ that generates an optical pulse which is subsequently phase modulated and temporally shaped with a pulse duration of 21 ns. This specially tailored pulse is then propagated via polarization maintaining (PM) fiber to a series of optical splitters and amplifiers. The splitters generate 48 amplified pulses of $1 \mathrm{n} J$ which are injected into 48 separate PAM's via PM fiber. Each PAM output is then split into four separate beams in the Preamplifier Beam Transport System (PABTS) and injected into the 192 main amplifier chains. The NIF subsystems: Master Oscillator Room (MOR), PAM and PABTS are combined into a system called the NIF front end or Optical Pulse Generation(OPG) system. Each of the 192 amplifiers, receives a $3 \mathrm{~J}$ injected pulse from the OPG. Below is a block diagram of the OPG location in NIF.

Each PAM consists of a diode pumped, solid state, Nd:glass regenerative amplifier ${ }^{(4)}$, a spatial beam shaping subsystem, and a flashlamp pumped, multi-pass amplifier ${ }^{(5)}$. The system gain of each preamplifier is $2 \times 10^{10}$ amplifying the $1 \mathrm{~nJ}$ master oscillator pulse to $14.7 \mathrm{~J}$ at the output of each PAM.

All PAM laser subsystems are mounted on a double sided vertical optical table housed in the optical support structure (OSS) which rolls on precision rails for rapid on-line installment. The regenerative amplifier and beam shaper are mounted on one side and feed light through a clearance hole to inject the 4pass amplifier on the other side of the optical table.

All PAM controls and diagnostics hardware occupy a separate area from the OSS in order to maintain optical cleanliness and thermal control. The electronics bay (e-bay) houses a VME based front end control system containing an embedded processor, general purpose analog and digital $\mathrm{I} / \mathrm{O}$, stepper motor control, and timing modules used to control PAM operation. Controlled hardware includes the pulser for the diode pumped solid state laser in the regenerative amplifier, 16 channels of stepper motor drive, electrical power and signal conditioning, ion pump controllers, and thermal heat exchangers for temperature control. A 30 $\mathrm{kJ}$ capacitor bank (PCU) is used to drive the flashlamps in the 4-pass amplifier is housed in a separate unit. Particular care has been devoted to electro-magnetic interference (EMI) and electrical isolation in the PAM and PCU. Below is a block diagram of the OPG subsystems.

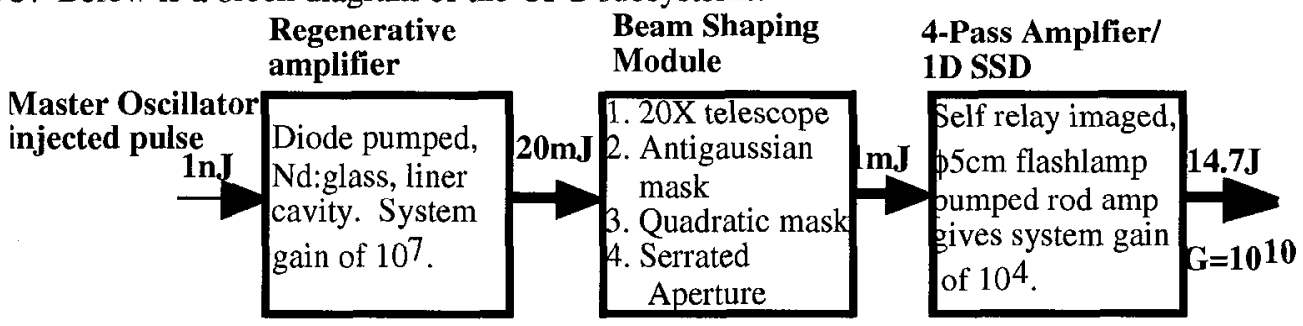

Figure 1. Block diagram of subsystems in all 48 PAM's. 
The OPG requirements are listed below and come from a flow down of the main laser requirements.

\begin{tabular}{|l|l|}
\hline Optical Pulse Generation Requirements & \\
\hline Injected energy into main amps: & $3.0 \mathrm{~J}$ \\
\hline -Preamp output energy (flattop beam) & $22 \mathrm{~J}$ \\
\hline -Preamp power energy (shaped beam) & $14.7 \mathrm{~J}$ \\
\hline Peak power at injection & $1.2 \mathrm{GW}$ \\
\hline Wavelength & $1053 \mathrm{~nm}$ \\
\hline Pulse duration & $20 \mathrm{~ns}$ \\
\hline Power balance (in 2 ns window) & $<3 \%$ \\
\hline Power dynamic range & $>125: 1$ \\
\hline Square-pulse-distortion & $<2.3$ \\
\hline Prepulse contrast & $>2 \times 10^{\wedge} 6$ \\
\hline Number of preamplifier modules & 48 \\
\hline
\end{tabular}

Table 1. General requirements for the $\mathrm{OPG}^{(1)}$

\subsection{DESCRIPTION}

\subsection{Regenerative amplifier}

The regenerative amplifier is the first stage of amplification in the PAM and represents the largest gain component in the NIF laser system.

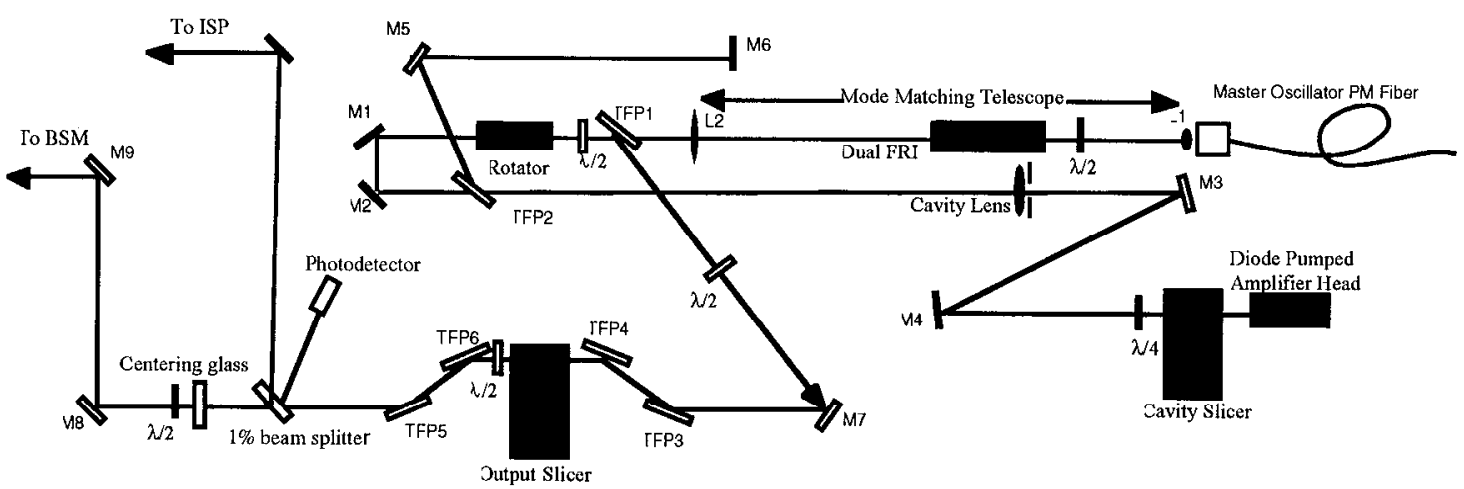

Figure 2. Optical layout of the regenerative amplifier used in the pre-amplifier module of the NIF. A single solid state amplifier is end pumped by a laser diode array and provides the required single pass gain of 1.6 per pass An injected beam is captured in the cavity for 27 passes, amplifying the beam energy from $1 \mathrm{~nJ}$ to a $23 \mathrm{~mJ}$ output. The beam is coupled out by means of polarization switching.

This regenerative amplifier is comprised of a folded, linear, $4.5 \mathrm{~m}, \mathrm{TEM}_{00}$ cavity using a diode-pumped, phosphate glass rod in an end-pumped configuration. A master oscillator pulse, is injected into the regenerative amplifier at the fiber launch. Refer to Figure 2 for the optical layout of the regenerative amplifier. Using both the fiber launch lens (L1) and a second lens (L2) we form a telescope that matches the spatial mode of the seed beam to the eigenmode of the $\mathrm{TEM}_{00}$ cavity. The seed also propagates through a dual stage Faraday isolator that protects the fiber from the much higher energy output leakage which propagates in the reverse direction. After the telescope, the beam propagates through a thin film polarizer (TFP1) which functions as the output coupler of the amplifier. The seed then propagates through a Faraday rotator and half wave plate that act as a directional coupler, separating the injected input from the regen output pulse Mirrors M1 and M2 are used to point and center the injected pulse into the cavity. The p-polarized seed is injected into the cavity through the thin film polarizer TFP2, goes through . a $3 \mathrm{~m}$ focal length cavity lens and mode limiting aperture. By adjusting the size of this aperture we force the 
cavity to operate in a single $\mathrm{TEM}_{00}$ spatial mode. Within the cavity the seed makes a Z-fold via mirrors M3 and M4, passes through the quarter waveplate the Q-switch Pockels cell (QS-PC) and into the amplifier head. It then reflects off the high reflectivity coating end of the rod reversing direction back through the cavity. With the QS-PC turned off, the polarization of the seed rotates 90 degrees from $\mathrm{P}$ to $\mathrm{S}$ due to double passing the quarter-waveplate and propagates back along its original path through the lens, aperture and reflects off of TFP2, propagating to mirror M5 and mirror M6. The mirrored end of the diode pumped amplifier rod and mirror M6 form the end mirrors of a linear cavity. Energizing the QS-PC traps the pulse in the cavity for $20-40$ round trips. When the pulse is amplified to the desired energy , the QSPC is switched off and the pulse is ejected from the cavity and reflects off of TFP1 and travels to the output slicer. The slicer PC is turned on long enough to pass the desired output pulse while eliminating unwanted prepulses that leak out of the regen. Following the output slicer, part of the beam is monitored with a photodiode. A motorized centering glass is then used to recover any alignment errors and mirrors M8 and M9 direct light to the beam shaping module (BSM.

\subsection{Beam shaping module}

The beam shaping module, located after the regenerative amplifier (regen), provides several functions. First, the $20 \mathrm{X}$ telescope, magnifies the Gaussian-shaped regen beam to over fill the shaping filters and apodizers that provide the desired shape injected into the 4-pass. Second, pixelated, chrome-on-glass filters spatially shape the beam to pre-compensate for the radial gain profiles of the main amplifiers, to provide a spatially flat-topped beam at target. Third, a serrated apodizer shapes the edge of the beam to minimize diffraction ringing and limit laser damage as the beam propagates through the rest of the system ${ }^{(\sigma)}$ Fourth, motorized stages provide rapid in situ placement of two different serrated apertures for different ICF missions. 'The output of the beam shaper is then folded to the other side of the table to be injected into the 4-pass amplifier. See Figure 3 below for a schematic of the BSM.

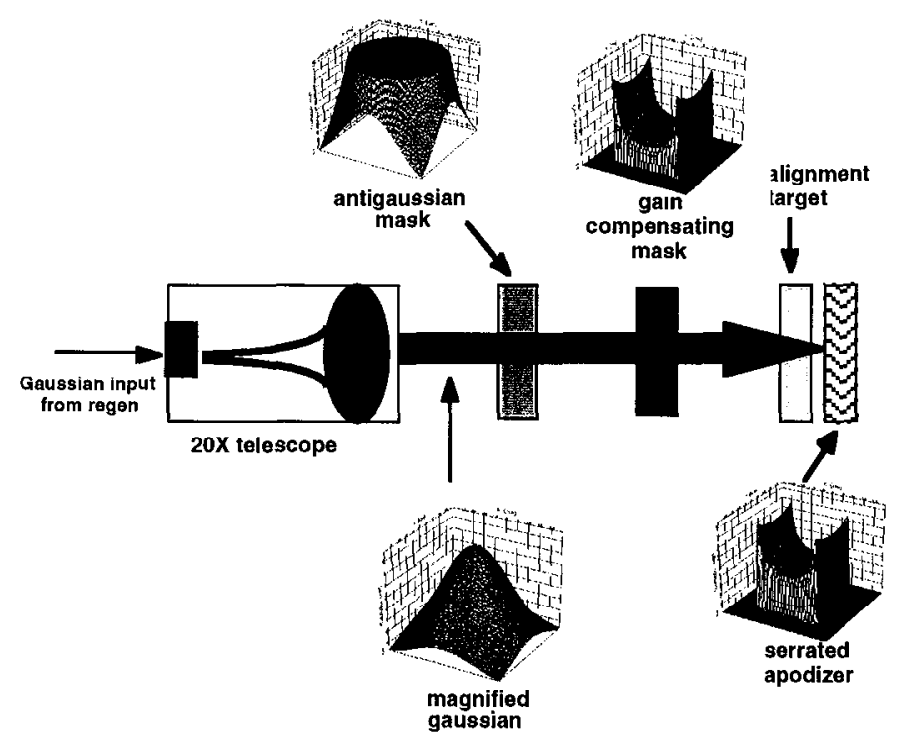

Figure 3. Diagram of beam shaping module used in NIF to expand, shape and provide $30 \mathrm{~mm} \times 30 \mathrm{~mm}$ beam to be injected into the 4-pass amplifier.

\subsection{4-pass amplifier/1D SSD}

The 4-pass amplifier is the second gain component in the PAM. This relay imaged, linear amplifier provides a gain of about $10^{4}$ as well as a user selectable, inter-cavity diffraction grating at Littrow for one dimensional smoothing by spectral dispersion (1D SSD). 


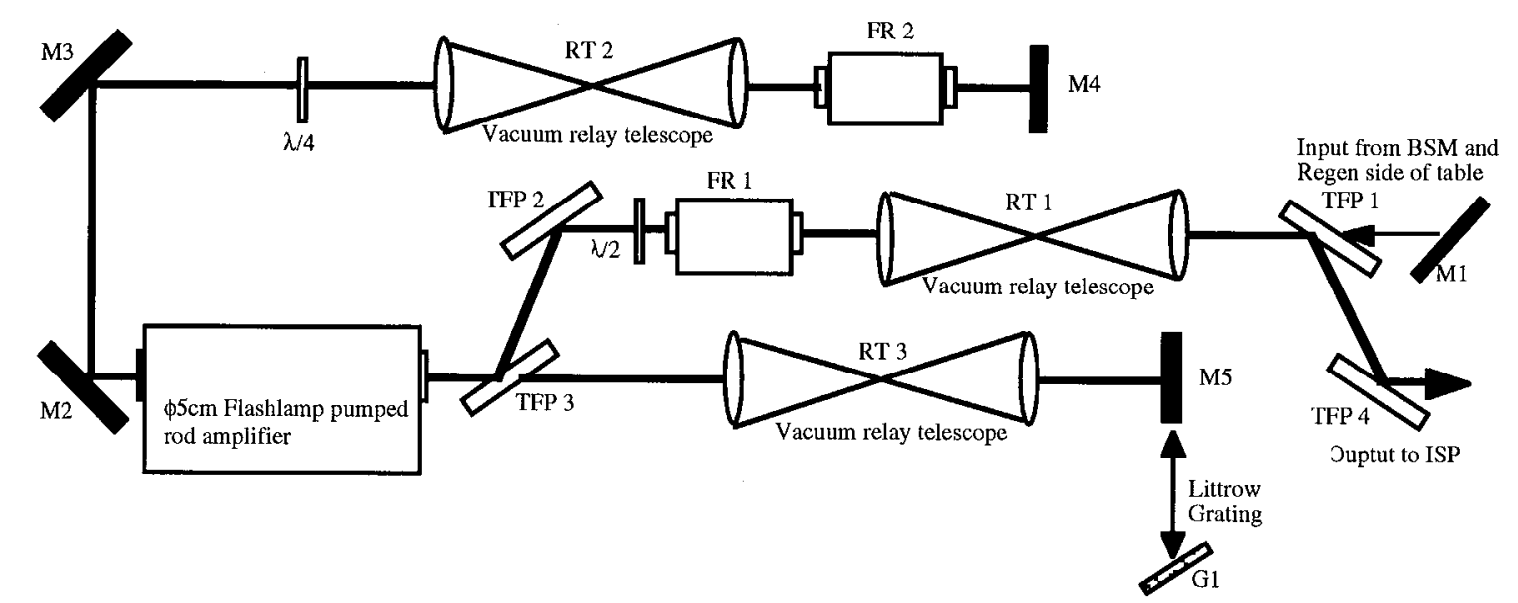

Figure Optical layout of flashlamp pumped 4-pass amplifier 14.7J is extracted in four passes giving a net gain of $10^{3}$.

The 4-pass amplifier is a passively switched linear amplifier which uses a 45 degree Faraday rotator, polarizers and a half waveplate to couple light into and out of the amplifier cavity providing four gain passes through the rod amplifier. A $\phi 5 \mathrm{~cm} \times 48 \mathrm{~cm}$ phosphate glass rod is pumped by 6 series flashlamps to provide the gain in the system. Three vacuum relay telescopes provide gain hold off, spatial filtering of high frequency components as well as relay imaging of the serrated aperture to prescribed locations in the cavity. The telescope, RT1, relays the serrated aperture to the center of the rod amplifier. The amplifier cavity is formed by the combination of mirrors, M4,M3, M2, M5, and telescopes RT2 and RT3. The image of the serrated aperture relayed to the center of the rod, is subsequently relayed to the cavity end mirrors, M4 and M5, using the cavity telescopes R'l'2 and R'l3. The quarter waveplate controls the polarization so the amplified pulse makes four passes through the rod, then out of the cavity via the two thin-film polarizers. The cavity Faraday rotator, FR2, cormpensates for thermal and stress-induced birefringence from the rod and vacuum spatial filters by rotating the noncircular polarization components by 90 degrees $^{(5)}$.

In all of the relay telescopes, each gain pass is separated by a field angle that helps isolate these passes to aid in holding off the gain which can cause parasitic oscillations. Following is a diagram of the pinhole configuration in the 4-pass telescopes.
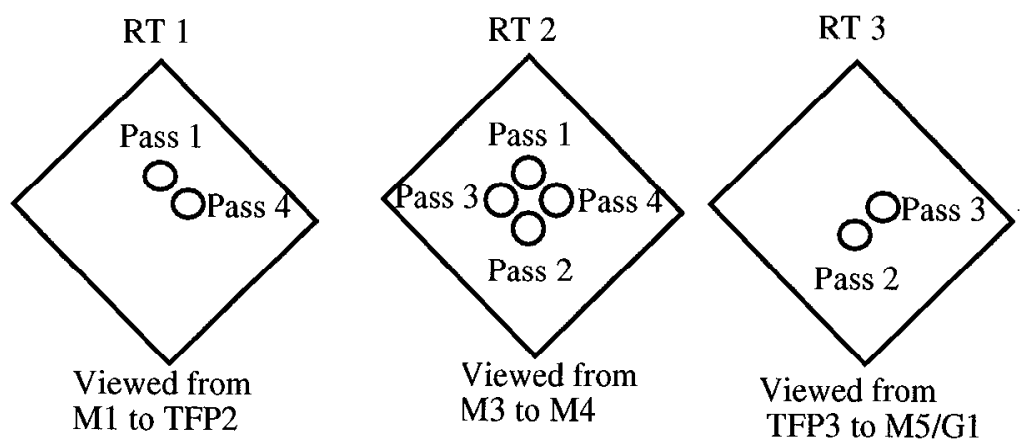

Figure 5. Pinhole configuration with field angle in the 4-pass cavity which helps with parasitic gain hold off.

\subsubsection{Regenerative amplifier}

\subsection{RESULTS}

Given the requirements for the entire OPG, we flow down these system requirements to the individual subsystems. Following is a list of requirements for the regenerative amplitier. 


\begin{tabular}{|c|c|}
\hline Output Energy & $20 \mathrm{~mJ}$ \\
\hline Square Pulse Distortion & $<1.45$ \\
\hline Energy Stability & $<3 \%$ \\
\hline Pre-pulse contrast & $10^{-6}$ \\
\hline Injected Energy & $<\operatorname{lnJ}$ \\
\hline
\end{tabular}

Table 2. NIF regenerative amplifier performance requirements.

The first energetics measurement made on the regenerative amplifier in the PAM was to evaluate the single pass gain of the diode pumped head. This will tell us if the regenerative amplifier has the gain and stored energy to meet the NIF requirements.

Using a cw Nd:YLF laser as a probe, we measured the double-pass gain of the end pumped rod amplifier as a function of pump diode driver current. The results are shown below along with a photo of the diode pumped head.

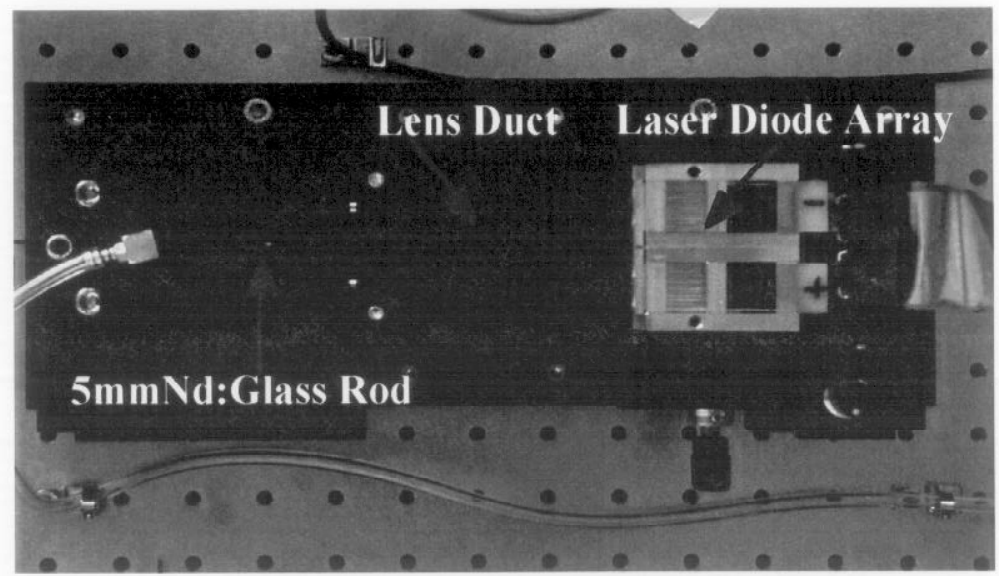

Figure . Photograph of diode pumped rod amplifier including: laser rod, laser diodes and lens duct.

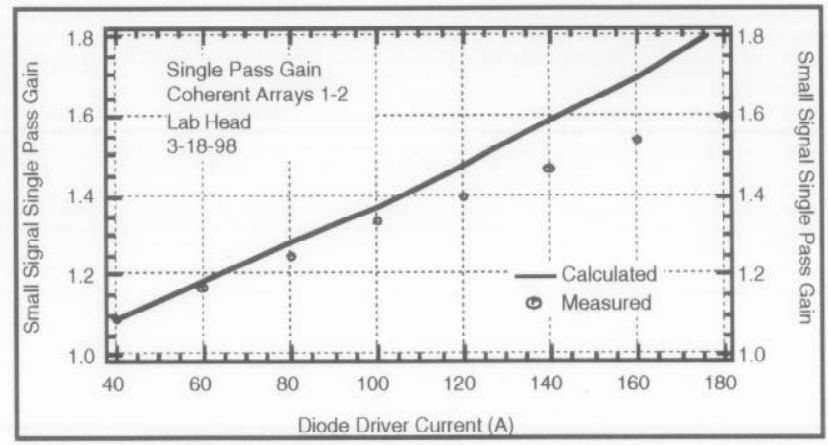

Figure. Experimental results show a single pass gain of 1.6 for the diode pumped head.

A simple model agrees with the data at the lower gains. At high gain the data rolls off due to ASE depleting the gain. The barrel of the diode pumped head is polished to support the TIR propagation of the pump light in the rod, but also supports the propagation of ASE which can itself extract energy from the rod.

One of the performance specifications of the NIF, is a precisely controlled temporal pulse shape at the target. A measure of temporal fidelity is square-pulse-distortion (SPD), which is the ratio of the gain at the leading edge of a square pulse to the gain at the end of the pulse, $S P D=G(0) / G(T)$. We measured SPD in

To determine the SPD of the regen, we use a high bandwidth (6Ghz) photoreceiver to measure the injected pulse and a second high bandwidth detector to temporally resolve the regen output pulse. These input and output pulses are converted to optical power and divided to show gain versus pulse length. The 
SPD is the ratio of the gain at the beginning of the pulse to the gain at the end of the pulse.

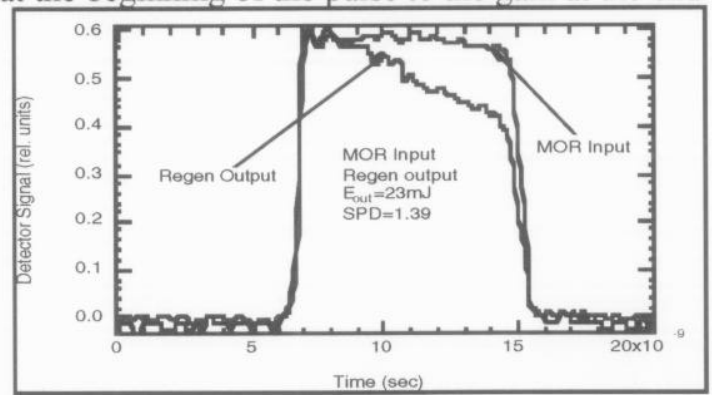

Figure 8. Results of SPD at a specific measurement shows PAM regen exceeds NIF requirements.

The plot above shows that the PAM regen exceeds NIF requirements given in Table 2. By injecting $<1 \mathrm{~nJ}$ from the master oscillator we extracted $23 \mathrm{~mJ}$ out of the regen with an SPD of 1.39 .

Following is a picture of the regenerative amplifier.

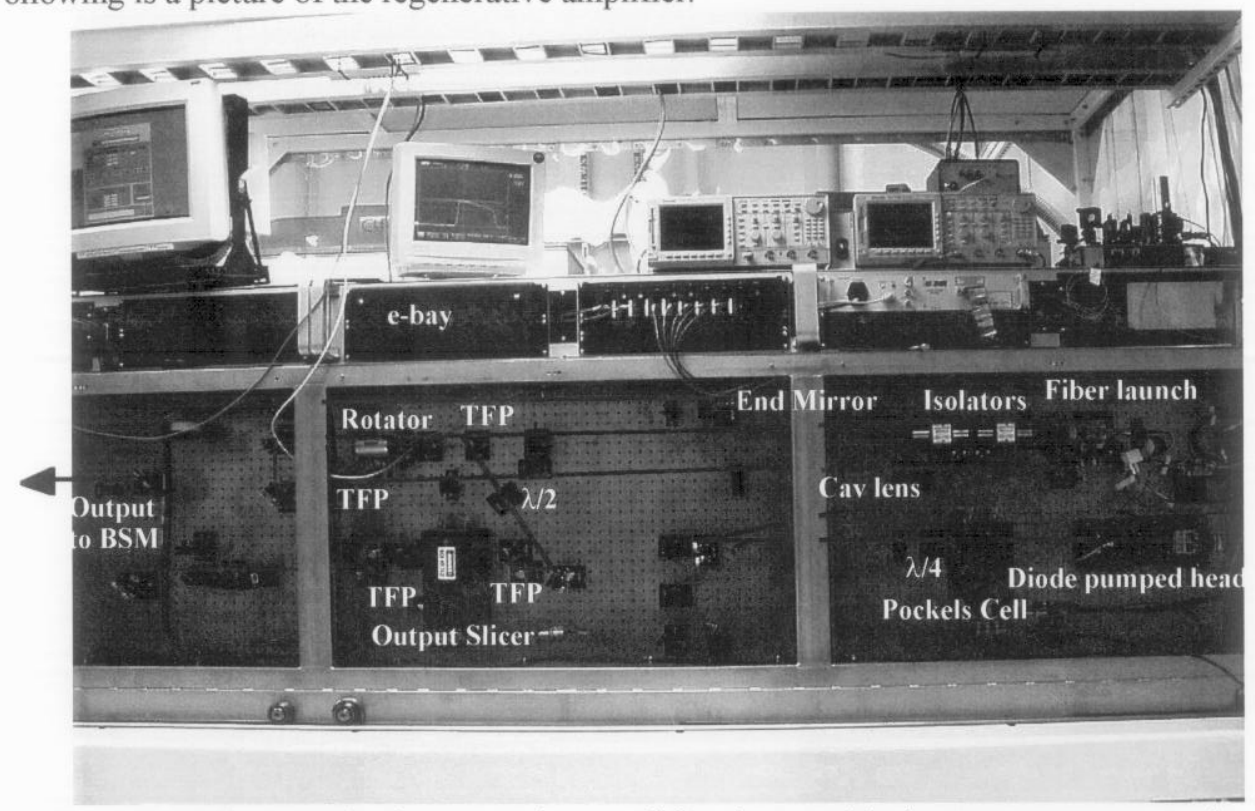

Figure 9. Photograph of regenerative amplifier shows critical components mounted on vertical laser table.

\subsubsection{Beam shaping module}

The beam shaping module (BSM) was designed and built by an outside contractor. As described above, this BSM mounted a 20X expanding telescope, two beam shaping masks, an alignment target and a serrated aperture. The BSM converts the Gaussian output of the regenerative amplifier into a $30 \mathrm{~mm} \mathrm{X}$ $30 \mathrm{~mm}$ square, apodized beam that is shaped to compensate for the spatial gain profiles of all the downstream amplifiers.. The serrated apertures and alignment masks are mounted on motorized stages to provide remote selection. . Below is a picture of the BSM mounted on the vertical table with camera images of the small Gaussian input and spatially shaped output, measured at the four-pass output. 


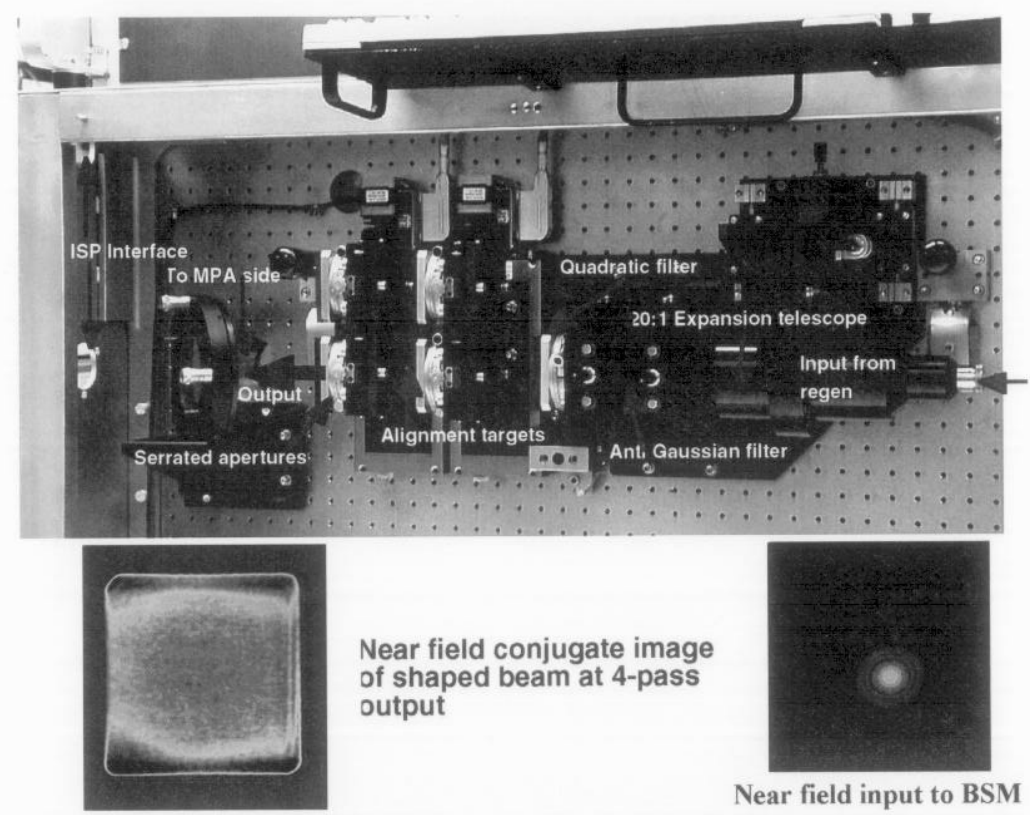

Figure 10. Beam shaping module provides a pre-shaped, $30 \mathrm{~mm}$ square beam from the regen, to be injected into the 4-pass pass amplifier.

\subsubsection{4-pass amplifier}

The last stage of amplification in the PAM is the 4-pass amplifier. A $30 \mathrm{mmX} 30 \mathrm{~mm}$ beam is injected into the relay imaged cavity and passed four times through a $\phi 5 \mathrm{~cm} X 48 \mathrm{~cm}$ phosphate glass amplifier rod. Below is list of requirements for the 4-pass amplifier.

\begin{tabular}{|c|c|}
\hline Output Energy & 22J (Flat-topped beam) \\
\hline & $14.5 \mathrm{~J}$ (Spatially Shaped) \\
\hline Peak Power & $8.8 \mathrm{GW}$ \\
\hline SPD (PAM/4-pass) & $<2.3 /<1.6$ \\
\hline Injection Energy & $1 \mathrm{~mJ}$ \\
\hline
\end{tabular}

Table 3. Energetics requirements list for the 4-pass amplifier.

In order to ensure the PAM would meet the energetics requirements, we characterized the $\phi 5 \mathrm{~cm}$ rod amplifier for small signal gain and radial gain profile. With the small signal gain and cavity transmission, we can predict the amount of energy to be extracted for a given injected energy. The spatial profile of the gain media is needed to design the spatial beam shaping masks that produce the required shape injected into the main amplifiers.

With a $\phi 10 \mathrm{~mm}$ probe beam, we measured the centerline gain of the amplifier head using two calorimeters. Below is a plot of the small signal gain versus delivered electrical energy and explosion fraction of the flashlamps.

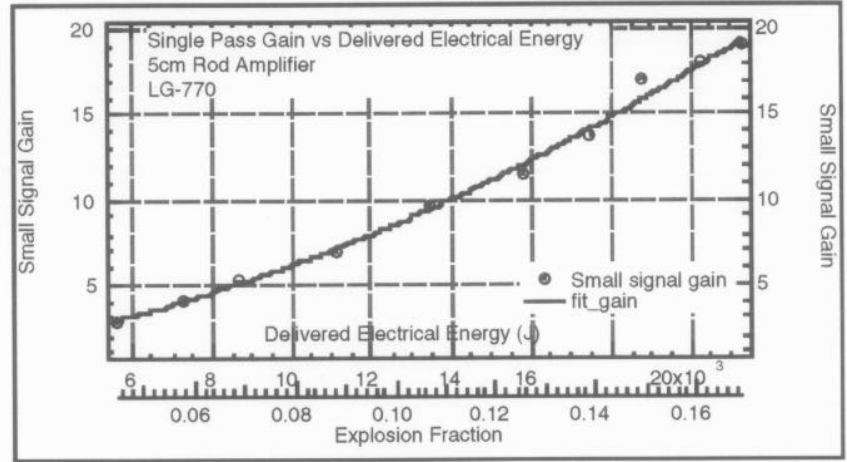

Figure 11. Results of measurements show a peak gain of 19. 
With a delivered electrical energy of $22 \mathrm{~kJ}$, we measure a single pass gain of 19 with an explosion fraction of 0.17 . This gain is sufficient to produce the energy requirements listed in table 3 .

We measured the radial gain profile by imaging the center of the rod at full aperture. The regenerative amplifier was used as the probe source and a reference image and gain image of the center of the rod were taken to determine the spatially dependent gain. Figure 12 shows the flat gain profile that we measured.

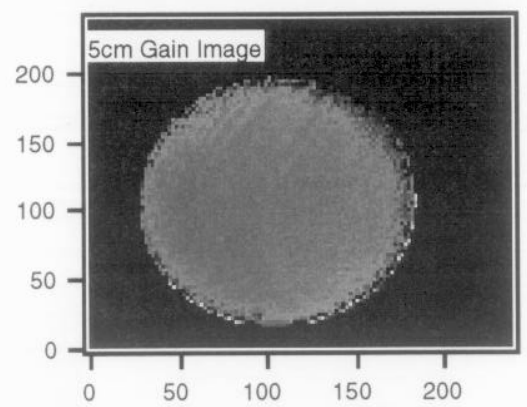

Figure 12. Radial gain profile measurement shows a relatively flat spatial gain profile.

Using the regenerative amplifier as an alignment source, the 4-pass amplifier was installed and aligned as shown in Figure 4. Figure 13 is a picture of the amplifier mounted on the OSS.

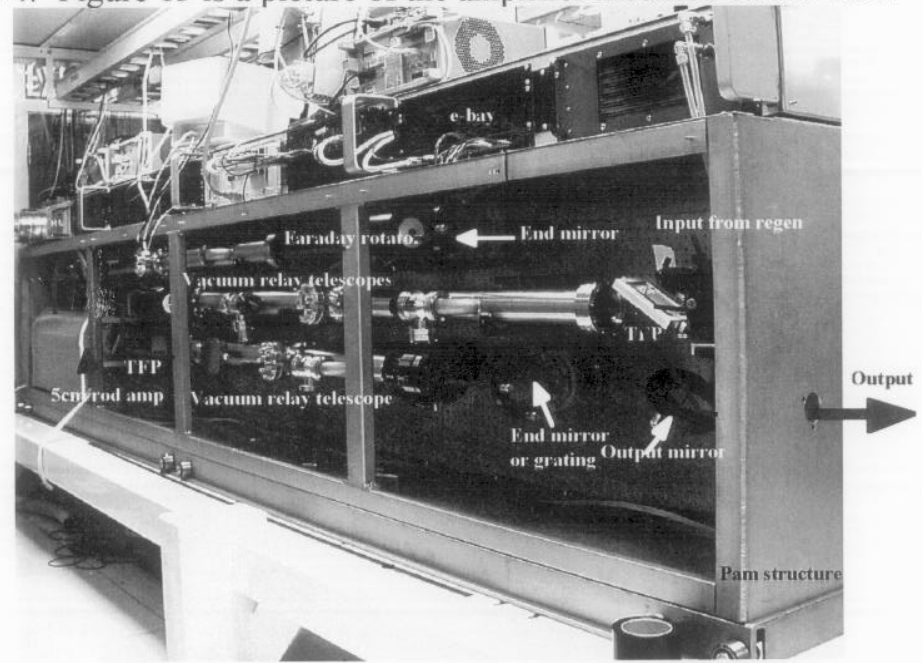

Figure 13. 4-pass amplifier is housed on one side of the optical support structure.

Once the 4-pass amplifier was installed and aligned, energy extraction measurements were conducted. Operating the $\phi 5 \mathrm{~cm}$ amplifier at peak gain, we varied the energy injected into the 4-pass amplifier and measured the output energy. The plot in Figure 14 shows energy extracted from the four-pass versus input energy. The solid line shows predicted energy based on a Frantz-Nodvik model ${ }^{(7)}$. 


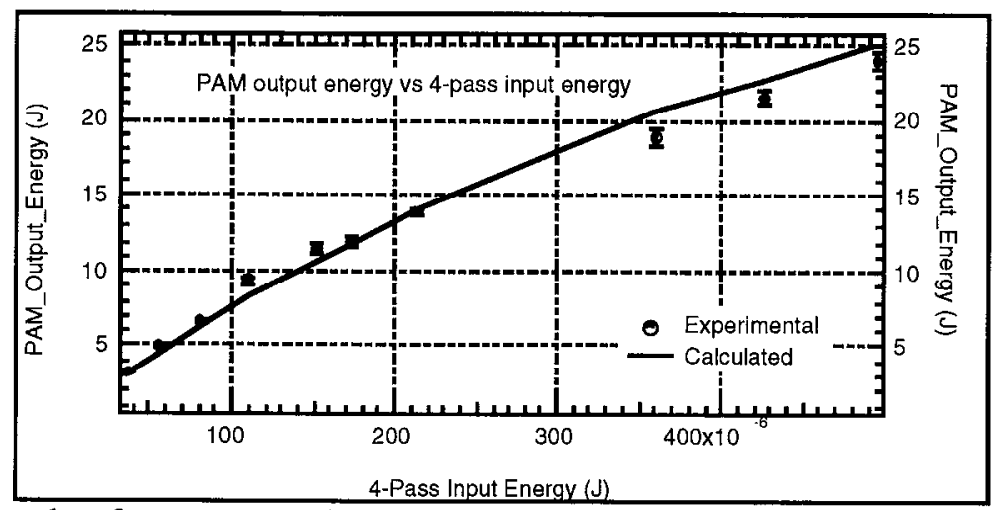

Figure 14. Results of energy extraction from 4-pass amplifier show the PAM exceeds the NIF OPG requirement of $22 \mathrm{~J}$ unshaped.

For an input energy of $488 \mu \mathrm{J}$ into the 4-pass cavity, we extracted $24 \mathrm{~J}$. This exceeds the $22 \mathrm{~J}$ requirement listed in Table 3 for an unshaped beam.

\subsection{CONCLUSION}

We have completed the construction and alignment of the high gain, Nd:glass NIF prototype preamplifier module. Using the general laser design developed on a physics testbed ${ }^{(1,2)}$ to meet NIF laser requirements, we have packaged the amplifier system in the optical support structure (OSS). This support structure is designed as a line replaceable unit(LRU). This LRU design provides rapid installment and removal to meet NIF shot rate requirements. Also, an electronics bay housed on the OSS provides controls and diagnostics. The PAM will remotely provide alignment for the laser system as well as define the level of energy injected into the main amplifiers which in turn defines energy delivered to target.

We have successfully demonstrated a fully inlegrated PAM prototype that exceeds NIF performance specifications for output energy, power and temporal pulse distortion. We continue to work to demonstrate the full performance requirements of the preamplifier-prototype before integrating with the OPG systems, MOR and PABTS, to demonstrate performance of the entire front end.

\subsection{ACKNOWLEDGMENTS}

Work done under the auspices of the U.S. Department of Energy by Lawrence Livermore National Laboratory under contract No. W-7405-Eng-48.

\subsection{REFERENCES}

1. J. K. Crane, R. B. Wilcox, N. W. Hopps, D. Browning, M. D. Martinez, B. Moran, F. Penko, J. E. Rothenberg, M. Henesian, C. B. Dane, and L. A. Hackel, "Integrated operations of the National Ignition Facility (NIF) Optical Pulse Generation Development System", Proc. $3^{\text {rd }}$ International Conf. On Solid State Lasers for Application to Inertial Confinement Fusion, Monterey, to be published, SPIE Proceedings Series, Bellingham, WA.

2. J. K. Crane. M. Martinez, B. Moran, C. Laumann, J. Davin, R. Beach, B. Golick, R. Jones, J.

Braucht, M. Perry, K. Skulina, F. Penko, S. Herman, S. Burkhart, S. Mitchell, " Description and performance of the preamplifier for the National Ignition Facility (NIF) laser system", Proc. $2^{n d}$ International Conf. On Solid State Lasers for Application to Inertial Confinement Fusion, Paris, France, 3047, pp. 601-609, SPIE Proceedings Series, Bellingham, WA, 1997.

3. S.C. Burkhart, R. Wilcox, D. Browning, F. Penko, "Amplitude and Phase Modulation with Waveguide Optics", Proc. 1st International Conf. On Solid State Laser for Application to Inertial Confinement Fusion, Monterey, 3047, p. 610-617, SPIE Proceedings Series Bellingham, WA, 1995

4. M.D. Martinez, J.K. Crane, L.A. Hackel, "Optimized, diode-pumped, Nd:Glass, Prototype Regenerative Amplifier for the National Ignition Facility", Proc. on Laser resonators, 3267, pp. 234-242, SPIE Proceedings Series Bellingham, WA 1998.

5. B.D. Moran, C.B. Dane, J.K. Crane, M.D. Martinez, F. Penko, L.A. Hackel, "Suppression of Paracitics and Pencil Beams in the High-Gain National Ignition Facility Multipass 
Preamplifier", Proc. on Optoelectronics and High-Power Lasers and Applications, 3264, pp 5664 SPIE Proceedings Series Bellingham, WA, 1998

6. M. Henesian, J. K. Crane, C. B. Dane, J. Davin, L. Kott, C. Laumann, J. Lawson, M. Martinez, B. Moran, J. Miller, R. Sacks, K. Skulina, W. Williams, "Diffraction modeling of the National Ignition Facility (NIF) Optical Pulse Generation (OPG) system and integration into the end-to-cnd system model", Proc. $3^{\text {rd }}$ International Conf. On Solid State Lasers for Application to Inertial Confinement Fusion, Monterey, to be published, SPIE Proceedings Series, Bellingham, WA.

7. L.M. Frantz, J. S. Nodvik, "Theory of Pulse Propagation in a Laser Amplifier", J. Appl. Physics, 64, 2346-2349, (1963). 